\title{
Multiphoton excitation of organic molecules in a cavity - superradiance as a
} measure of coherence

Inga S. Ulusoy, ${ }^{1, \text { a) }}$ Johana A. Gomez, ${ }^{1}$ and Oriol Vendrell ${ }^{1, b}$

Theoretical Chemistry, Institute of Physical Chemistry, Heidelberg University, Im Neuenheimer Feld 229, 69120 Heidelberg, Germany

(Dated: 22 October 2020)

Coherent excitation of a molecular ensemble coupled to a common radiation mode can lead to the collective emission of radiation known as superradiance. This collective emission only occurs if there is an entanglement between the molecules in their ground and excited state and can therefore serve as a macroscopic measure of coherence in the ensemble. Reported here are wave packet propagations for various pyrazine models of increasing complexity and molecular ensembles thereof. We show that ensemble coherence upon photoexcitation can prevail up to relatively long time scales, although the effect can diminish quickly with increasing ensemble size. Coherence can also build up over time and even reemerge after the molecules have passed through a conical intersection. The effect of the pump-pulse characteristics on the collective response of the molecular ensemble is also studied. A broad-band pulse imprints a large amount of initial coherence to the system, as compared to a longer pulse with a smaller spread in the frequency domain. However, the differential effects arising from a different pulse duration and coherent bandwidth become less prominent if the emission of light from the ensemble takes place after a non-adiabatic decay process.

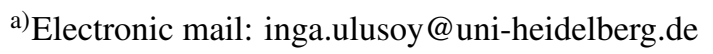

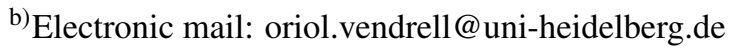


Multiphoton excitation of a cavity-molecular ensemble

\section{INTRODUCTION}

In the presence of a quantized electromagnetic mode, molecular states can hybridize with the light mode forming light-matter states, the lower polariton (LP), upper polariton (UP) and darkstate polariton (DSP). ${ }^{1}$ In the case of several quanta of excitation in the ensemble, a collective, and thus coherent, spontaneous emission (superradiance (SR) or superfluorescence) ${ }^{2-5}$ through the matter part of the ensemble can be triggered. The intensity of the emitted radiation is larger by a factor of $N$ compared to an independent simultaneous spontaneous emission of $N$ emitters (atoms/molecules), ${ }^{2,6-8}$ and opens up new applications with respect to unconventional laser architectures, quantum networks and information storage. ${ }^{9-13}$ In addition to the increased emission intensity, the duration of the emission is also shorter at $\tau_{S R}=\tau_{0} / N$ (see [4,7], with $\tau_{0}$ the half-time of an independent emitter) resulting in a short burst of radiation.

SR is the result of a correlation build-up in the sample, which is facilitated through the coupling of the emitters to a common radiation mode. Initially, the emitters emit photons independently, and after a lag time that signifies (1) the build-up of correlation and (2) reaching a regime where about half of the sample has emitted a photon, an SR burst is observed. ${ }^{14}$ After the burst, the emission continues independently. As SR is a result of symmetry - the indiscernibility of the emitters - inhomogeneous broadening affects the SR characteristics, for example, requiring a threshold number of particles $N_{c}$ for SR to manifest. ${ }^{4,14}$ Inhomogeneous broadening effectively results in an emission from levels with slightly different frequencies, and can also lead to subradiance (much longer decay times), SR beats, SR ringing, or SR quenching. ${ }^{15}$

We investigate here the role of the pump-pulse characteristics preparing the initial ensemble state, as well as the subsequent vibronic dynamics of the molecules, in shaping the emission of radiation to a quantized cavity mode coupled to the molecular ensemble. Inhomogeneous broadening is not taken into account, as neither are direct intermolecular interactions. However the latter may increase cooperativity significantly as they can reduce the inhomogeneous broadening (interaction-induced synchronization). ${ }^{7,16} \mathrm{SR}$ is a transient phenomenon, and we identify it by the ensemble-size scaling of the time-dependent emission rate.

Previously, SR characteristics have been investigated theoretically by several authors - for example, investigating the role of inhomogeneous broadening, ${ }^{6,15}$ or the impact of the cavity decay rate, ${ }^{17,18}$ and in the presence of dissipation. ${ }^{19}$ A plasmonic Dicke effect in analogy to SR has also been found ${ }^{20,21}$ and hence SR is not limited to electronic transitions. ${ }^{22}$ As SR is a result of the 
quantum coherence of the emitters, it provides a privileged window by which this coherence can be observed experimentally. The coherence in molecular dimers has been shown to depend on their spatial separation, ${ }^{23}$ as the locally different environment leads to detuning and a loss of entanglement; but generally, the coherence is dominated by the vibrational part of the wave function - the vibronic state - so while the local environment plays a role, even more important are the Huang-Rhys factors characterizing the different electronic states. The emergence and evolution of vibronic coherence as a function of the preparation step can be established through the comparison of different laser-pulse lengths and spectral bandwidths, and has implications for experiments using narrow-width laser pulses that attempt to reproduce processes initiated by e.g. sunlight. ${ }^{24}$

In the following, the Hamiltonian and calculated observables are introduced; followed by wavepacket propagations for pyrazine models of increasing complexity, and ensembles thereof, as an exemplary organic heteroaromatic molecule that exhibits internal dissipation channels. ${ }^{25,26}$

\section{THEORY}

The ensemble-cavity Hamiltonian for $N$ molecules coupled to a cavity mode reads:

$$
\hat{H}=\sum_{m=1}^{N} \hat{H}_{\mathrm{mol}}^{(m)}+\hat{H}_{\mathrm{cav}}+\hat{H}_{\mathrm{las}}
$$

with $\hat{H}_{\mathrm{mol}}^{(m)}$ the Hamiltonian for the $m$-th molecule, $\hat{H}_{\mathrm{cav}}$ the cavity and cavity-molecule coupling Hamiltonian, and $\hat{H}_{\text {las }}$ the laser field (treated classically). The cavity mode is described as a single quantized electromagnetic mode with a constant electric field amplitude across all members of the molecular ensemble,

$$
\hat{H}_{\mathrm{cav}}=\left(\hbar \omega_{C}+\frac{1}{2}\right) \hat{a}^{\dagger} \hat{a}+g \vec{\varepsilon}_{c} \overrightarrow{\hat{D}}\left(\hat{a}^{\dagger}+\hat{a}\right)+\frac{1}{2}\left(g \vec{\varepsilon}_{c} \overrightarrow{\hat{D}}\right)^{2}
$$

where $\vec{\varepsilon}_{c}$ is the cavity mode polarization direction, $\overrightarrow{\hat{D}}=\sum_{m}^{N} \vec{\mu}^{(m)}$ the dipole operator for the $N$ molecules with dipole moment $\vec{\mu}$, and $g=\sqrt{\frac{\hbar \omega_{C}}{2 V \varepsilon_{0}}}$ the coupling strength of the electromagnetic mode or cavity-mode field strength. The laser interaction term reads

$$
\hat{H}_{\mathrm{las}}=-\vec{E}(t) \vec{D}
$$

with electric field $\vec{E}(t)=\vec{\varepsilon}_{L} A(t) \cos \left(\omega_{L} t\right)$, where $\vec{\varepsilon}_{L}$ is the laser field polarization direction, $A(t)$ is the electric field envelope and $\omega_{L}$ the carrier frequency of the laser. Without loss of generality 
we make the model assumption that the laser field couples directly to the molecules and not to the cavity. $^{27}$ The length gauge and the dipole approximation are used for both the quantized cavity mode and the laser field. As in previous works ${ }^{28,29}$, the molecular Hamiltonian corresponds to the four-dimensional vibronic coupling Hamiltonian for pyrazine. ${ }^{25,26}$

\section{A. Quantum dynamics propagation and analysis}

The time-dependent Schrödinger equation for the molecular ensemble-cavity wave function is propagated using the MCTDH method ${ }^{30,31}$ in its multilayer generalization ${ }^{32-34}$ with the Heidelberg MCTDH package. ${ }^{35}$

The laser intensity is tuned such as to achieve a certain amount of population of the $\mathrm{S}_{2}$ excited electronic state for a single molecule, for example 50\%. For different ensemble sizes, the laser intensity is then kept constant at this value, which immediately results in absorption in the multi-photon regime through coherent absorption from all members of the ensemble. Specific pulse parameters are provided in Table I. Alternatively, the initial ensemble state can be prepared assuming an instantaneous vertical excitation, either as a superposition of the $\mathrm{S}_{0}$ and $\mathrm{S}_{2}$ electronic states for all molecules, or as a pure exited state with all molecules instantaneously populating the $\mathrm{S}_{2}$ state. In both situations the vibrational wavefunction at $t=0$ corresponds to the vibrational ground state on the $\mathrm{S}_{0}$ PES (cf. Eq. 8).

Through the wave packet propagations we gain access to the cavity photon number expectation value, $\left\langle N_{\mathrm{Ph}}\right\rangle(t)$ :

$$
\left\langle N_{\mathrm{Ph}}\right\rangle(t)=\left\langle\Psi(t)\left|\hat{a}^{\dagger} \hat{a}\right| \Psi(t)\right\rangle
$$

which provides a direct measure of the total amount of radiation emitted by the molecular ensemble into the cavity mode. The populations $P_{j}^{(m)}(t)$ signify the single-molecule electronic state populations for the $j$-th electronic state, obtained by projecting onto the single-molecule electronic states $\psi_{j}^{(m)}$. For example, for the $\mathrm{S}_{0}$ state

$$
P_{0}^{(m)}(t)=\left|\left\langle\psi_{0}^{(m)} \mid \Psi(t)\right\rangle\right|^{2}
$$

where $|\Psi(t)\rangle$ is the time-dependent state of the whole ensemble and cavity at time $t$. Because the $P_{j}^{(m)}(t)$ populations are one-molecule properties and all molecules are identical, the $m$ index becomes irrelevant and can be dropped. 


\section{B. Superradiance and stimulated emission}

SR is a result of symmetry, and can be understood as the enhanced emission of an ensemble after the build-up of correlation between the members of this ensemble. When about half of the ensemble is in an excited state, and the other half in the ground state; and the members of the ensemble are indistinguishable, the ensemble is in a symmetry-entangled superposition state where the excitation can reside on either member of the ensemble (Dicke state). ${ }^{2}$ From this superposition state, the rate of emission towards the completely de-excited ensemble is $N$ times as large compared to $N$ independent emitters, as there are $N \times N$ possibilities of realizing the de-excitation pathway. The electromagnetic mode acts as a facilitator in that it provides the means to reach this highly symmetric state through the orientation of the (atomic/molecular) dipoles. The coupling to the field needs to be identical for each emitter, as inhomogeneities break the symmetry. In the following, we do not consider long-time processes such as emitter loss, background fluctuations and photon loss through the cavity.

The short-time dynamics of the ensemble, where the reference time $t_{\text {ref }}$ corresponds to the onset of resonance with the cavity, can be approximated as ${ }^{8}$

$$
\begin{aligned}
\left\langle N_{P h}\right\rangle(t) & =t^{2} \mu_{g e}^{2} g^{2}\left(\sum_{m}^{N} P_{e}\left\langle\phi_{e}^{(m)} \mid \phi_{e}^{(m)}\right\rangle+\sum_{m=1}^{N} \sum_{\substack{n=1 \\
n \neq m}}^{N} P_{g} P_{e}\left\langle\phi_{e}^{(m)} \mid \phi_{g}^{(m)}\right\rangle\left\langle\phi_{g}^{(n)} \mid \phi_{e}^{(n)}\right\rangle\right) \\
& =t^{2} \mu_{g e}^{2} g^{2}\left(N P_{e}+\left(N^{2}-N\right) P_{g} P_{e}\left|S_{g e}\right|^{2}\right) .
\end{aligned}
$$

where the rotating wave approximation (RWA) and a second-order expansion of the time-evolution operator have been used. Here, $\mu_{g e}$ is the transition dipole moment between the electronic ground $g$ and excited $e$ states and is identical for all molecules; the populations $P_{g}$ and $P_{e}$ are the singlemolecule electronic state populations for electronic states $g$ (ground) and $e$ (excited) with electronic wavefunctions $\psi_{g}^{(m)}$ and $\psi_{e}^{(m)}$ for molecule $m$ (see above). The nuclear wave packets, $\phi_{g}^{(m)}$ and $\phi_{e}^{(m)}$, are independently evolving on the electronic ground and excited states. The overlap integral $S_{g e}^{(m)}=\left\langle\phi_{g}^{(m)} \mid \phi_{e}^{(m)}\right\rangle$ over the nuclear wave packets of the different electronic states in the first line of Eq. 6 is equal for all molecules, resulting in the expression of the second line. For atoms, this overlap is always equal to 1 . In principle, the overlap integral would be different for the different molecules in the case of inhomogeneities, but this possibility is not being considered here. Importantly, the loss of coherence of the nuclear wave packets evolving on the ground and excited electronic states, resulting in a decrease of $\left|S_{g e}\right|^{2}$, will lead to a diminished coherent photon 
emission, whereas this has no effect on the linear term proportional to $P_{e}$.

The above expression Eq. 6 is valid for an initial wave function $\left|\Psi\left(t_{\text {ref }}\right)\right\rangle$ with zero photons in the cavity $\left(\left\langle N_{P h}\right\rangle\left(t_{\text {ref }}\right)=0\right)$ at the reference time, and with the molecular part given by a Slater product of molecular wavefunctions

$$
\left|\Psi\left(t_{\text {ref }}\right)\right\rangle=\prod_{m=1}^{N}\left(\sqrt{P_{g}} \phi_{g}^{(m)}\left(Q_{m}\right)\left|\psi_{g}^{(m)}\right\rangle+\sqrt{P_{e}} \phi_{e}^{(m)}\left(Q_{m}\right)\left|\psi_{e}^{(m)}\right\rangle\right)|0\rangle
$$

where, for completeness, the dependence of the nuclear wave packets on the nuclear coordinates $Q_{m}$ is explicitly noted. This situation is fulfilled after the preparation by the laser pulse and, in any case, before the onset of the interactions with the cavity, which entangle the molecules destroy the product form of the wavefunction. Any more-than-linear scaling of the radiation rate with the number of molecules is only possible through the presence of coherence between the electronic states of the molecules ${ }^{2}$.

We introduce now the time-dependent emission rate $k_{e m}(t)$ defined as the derivative of the cavity photon number expectation value:

$$
k_{e m}(t)=\frac{d}{d t}\left\langle N_{\mathrm{Ph}}\right\rangle(t)
$$

As emission takes place in the transient regime following the photoexcitation by a laser, the rate is dependent on time. This emission rate can be normalized by the emission rate for one isolated molecule, $k_{e m}^{N=1}(t)$

$$
k_{e m}^{r e l}(t)=k_{e m}^{N}(t) / k_{e m}^{N=1}(t)
$$

to reveal the scaling of the emission rate with $N$. Note that it is not discriminated here from which polariton mode the excited-state cavity population is inherited, and that the ensemble carries multiple excitations.

In experiments, the sample can undergo inhomogeneous broadening leading to dephasing; for SR to manifest, the dephasing time needs to be shorter than the delay time of the SR burst. ${ }^{6}$ In this work, all molecules are assumed perfectly aligned, and no mechanism inducing inhomogeneous broadening such as collisions, Doppler effects, and slightly different orientations or distribution of initial rovibrational states is considered. We thus study an artificially homogeneous system. However, besides the homogeneous loss of coherence directly manifest in the overlap factor $\left|S_{g e}\right|^{2}$, there is a further factor contributing to the amount of coherence, namely the duration of the excitation process by the laser pulse. During the interaction with the laser pulse, the system is 
Multiphoton excitation of a cavity-molecular ensemble

continuously pumped to the excited state; thus, there are portions of the wave packet that reach the excited state earlier than others and can already undergo further dynamics. Finally, portions of the wavepacket excited at different times jointly contribute to the emission of light to the cavity, making the SR process sensitive to the preparation step, i.e. to the coherence of the light field exciting the ensemble.

\section{RESULTS AND DISCUSSION}

In the following, the wave packet dynamics after absorption of several photons by the ensemble is investigated. Each pyrazine molecule can at most absorb one photon, resulting in a population transfer from $S_{0}$ to $S_{2}$; from the $S_{2}$ electronic state, the molecule undergoes rapid internal conversion (IC) resulting in population of the $S_{1}$ electronic state. We investigate different scenarios:

- pyrazine as a two-level system with the IC channel closed - in this case, the only channel leading to a depopulation of $S_{2}$ is the radiative emission of the molecules into the cavity, $S_{1}$ is not populated;

- pyrazine with the cavity resonantly coupling $\mathrm{S}_{0}$ and $\mathrm{S}_{2}$ at (i) the Franck-Condon (FC) point and (ii) the $S_{2}$ potential minimum;

- pyrazine with the cavity resonantly coupling $S_{0}$ and $S_{1}$ at (i) the FC point and (ii) the $S_{1}$ potential minimum.

The first scenario exemplifies the emergence of SR and the role of the tuning modes - the pure vibrational dephasing - in the short-time regime. In the second scenario, the role of laser-pulse duration and spectral width is investigated, with focus on the short- and long-lived coherence. Lastly, we approach the question of whether coherence can manifest after a process such as IC through a conical intersection (CI), and whether SR could be utilized in frequency conversion.

\section{A. Model without internal conversion: the effect of pure vibrational motion}

Initially, an ensemble of a reduced pyrazine model is studied where the IC channel that leads to nonradiative population transfer from $S_{2}$ to $S_{1}$ is closed by setting the nonadiabatic coupling constant between these two states to zero. The excitation in the ensemble can either be generated by instantaneous transfer of population to the $S_{2}$ electronic state from the vibrational ground state 
on the $\mathrm{S}_{0}$ PES; or, by utilizing a laser pulse (with pulse full-width at half maximum FWHM of $\tau_{L}=$ $18 \mathrm{fs}$, intensity $E_{0}=1.26 \cdot 10^{12} \mathrm{~W} / \mathrm{cm}^{2}$ and frequency $\omega_{L}=4.7233 \mathrm{eV}$ ), leading to a continuous population transfer. The cavity couples $S_{0}$ and $S_{2}$ resonantly at the $\mathrm{FC}$ point $\left(\omega_{C}=4.7233 \mathrm{eV}\right)$. Three different coupling strengths are investigated, with the lowest coupling strength (w) leading to a Rabi splitting of $\Omega_{R}^{(w)}=0.02 \mathrm{eV}$, an intermediate coupling strength (m) resulting in $\Omega_{R}^{(m)}=$ $0.13 \mathrm{eV}$, and the highest coupling strength (s) with $\Omega_{R}^{(s)}=0.28 \mathrm{eV}$ for one molecule. Two sets of calculations are carried out: First, the coupling constant $g$ is not scaled by $1 / \sqrt{N}$, resulting in an increased Rabi splitting with increasing number of molecules, and a higher cavity-molecule interaction strength for larger $N$; and second, the coupling constant is scaled by $1 / \sqrt{N}$, resulting in a constant Rabi splitting and interaction strength, but decreased effective cavity-molecule coupling per individual molecule for increasing $N$. The first case corresponds to an increased emitter density in the cavity, while in the second case the emitter density is kept constant. The first scenario is typically realized in SR experiments, resulting in the well-known SR signature $N^{2}$ in the intensity scaling. The second scenario has been studied previously ${ }^{19}$ and has been found to result in a linear scaling of the intensity with $N$ for SR, which also becomes apparent from appropriately substituting the $1 / \sqrt{N}$ scaling of $g$ in Eq. 6 .

For an instantaneous excitation, the SR characteristics are determined through the short-term response of the ensemble to the excitation (at $t_{\text {ref }}=0$ ) and are shown in Fig. 1a) with no rescaling of $g$ over ensemble sizes, and Fig. 1b) with rescaled $g$. The rates in Fig. 1a) scale linearly with the number of molecules for an equal number of photons and molecules in the ensemble (dot markers), i.e. all molecules are initially excited with $100 \%$ probability, but scale quadratically when a coherent $50 \%$ excitation is present in the ensemble $\left(N_{p h}=N / 2\right.$, x markers). An analytic linear and quadratic scaling are included in the plot to guide the eye. The magnitude of the cavitymolecule coupling strength has nearly no influence on the relative emission rate dependence on $N$, as the curves for $(\mathrm{w}),(\mathrm{m})$ and (s) align almost perfectly. Note that the absolute rate is different, though, for the different coupling strengths, with a higher rate for the more strongly coupled cavity (by a factor of $\approx 200$ ).

In Fig. 1b), the relative emission rate for the same model system is shown, but here the overall cavity-molecule interaction remains constant, so that the effective coupling for each molecule decreases. For an equal number of photons and molecules in the ensemble, the relative emission rate remains constant (only shown for $(\mathrm{m})$ ). The SR emission, that is observed for $50 \%$ excitation present in the cavity $\left(N_{p h}=N / 2\right)$, now carries the signature of a linear scaling with $N$, as discussed 

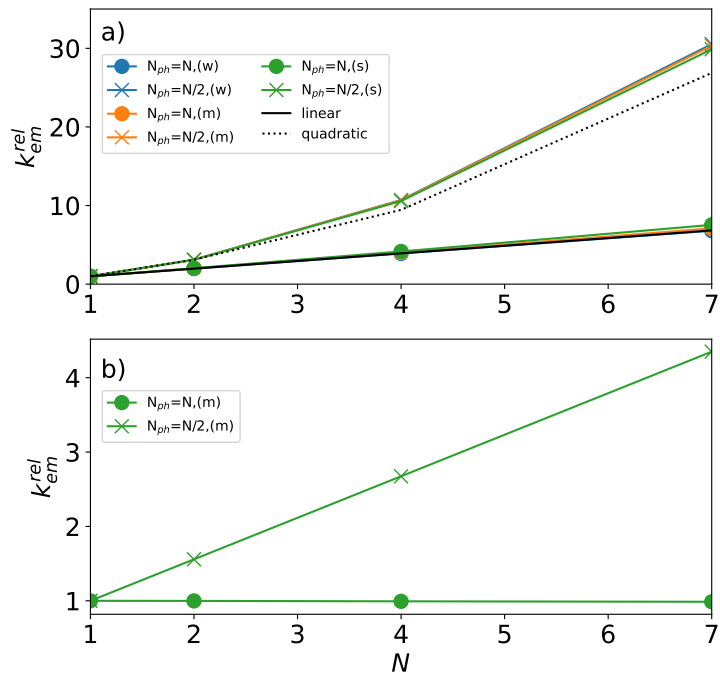

FIG. 1. Relative emission rate at time $t_{\text {ref }}=0$ over number of molecules $N$ in the cavity for the pyrazine model with the IC channel closed. The overall photon number in the ensemble $N_{p h}$ is either equal to $N$ or $N / 2$; a) $g$ is not rescaled by $1 \sqrt{N}$ and three different cavity-molecule coupling strengths (w), (m), and (s) are shown; b) $g$ is rescaled by $1 \sqrt{N}$ and the cavity-molecule coupling strength is (m).

above. In the following, the cavity-coupling constant is scaled by $1 / \sqrt{N}$, so that the SR signature corresponds to a linear dependence of the relative emission rate on $N$. We focus on the cavitymolecule coupling strength $(\mathrm{m})$ with a constant Rabi splitting of $\Omega_{R}^{(m)}=0.13 \mathrm{eV}$ for all cases studied hereafter.

In Fig. 2, the populations of the electronic ground and excited states are shown for a), b) laserpulse excitation and c), d) $50 \%$ instantaneous excitation. First, we investigate the impact of the pure vibrational motion on the photoemission process by essentially transforming the model into an atom-like two-level system (here, the vibrational modes are harmonic oscillators and the potentials of $S_{0}$ and $S_{2}$ are perfectly aligned at the FC point - this is achieved by setting all the tuning parameters (Huang-Rhys factors) in the pyrazine model to zero). ${ }^{28,29}$ The ensuing dynamics for the atom-like system are shown in b) and d), while in a) and c) the anharmonic character of the vibrational modes is retained as in the original pyrazine model, with the minimum of $S_{2}$ not at the FC point. Comparing a) and c), laser-pulse excitation vs. instantaneous excitation, the dynamics evolve more rapidly in the case of c), but the overall characteristics are similar. For c) and d), instantaneous excitation with and without vibrational tuning, it is apparent that the vibrational tuning leads to a strong damping of the Rabi oscillation in c), while the Rabi cycling is very prominent in 
Multiphoton excitation of a cavity-molecular ensemble

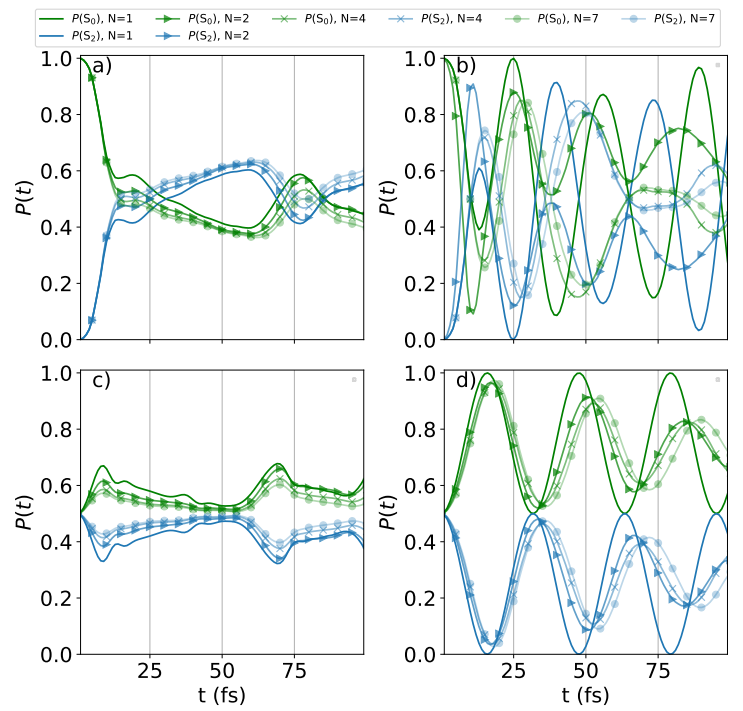

FIG. 2. The pyrazine model with the IC channel closed, population of the electronic states. Excitation with a) laser pulse, b) laser pulse (no vibrational tuning in the molecule), c) instantaneous excitation, d) instantaneous excitation (no vibrational tuning in the molecule).

d) with a cycle length of $32 \mathrm{fs}$. Interestingly, with increasing molecule number, the Rabi oscillations also appear damped in d). This is a consequence of higher excitations in the cavity $\left(\mathrm{N}_{p h}>1\right)$. It appears that on the longer time-scale, the cavity-mode population impacts the wave-packet dynamics through the Rabi cycling and leads to a similar damping as the vibrational modes of the molecule, but to a lesser extent. This effect is more pronounced after laser-pulse excitation, b). The situation in b) and d) can be depicted as a set of coherently excited spin systems coupled to a single bosonic mode, as opposed to the spin-boson model, where a bosonic bath is coupled to a single spin. The cavity-mode populations are shown in the Supporting Information (SI).

The corresponding time-dependent emission rates are shown in Fig. 3a)-d). While the emission rate in a) and c) is similar, with c) slightly more rapid dynamics as a result of a lesser spread of the excited wave packet in time, the emission rates in b) and d) exhibit more frequent, but in magnitude decreasing, recurrences. Comparing with Fig. 2, these recurrences appear when the population of $S_{0}$ and $S_{2}$ is roughly 0.5 . The peaks at a) $12 \mathrm{fs}$ and $72 \mathrm{fs}$ and c) $4 \mathrm{fs}$ and $64 \mathrm{fs}$ exhibit linear scaling with $N$ and correspond to SR emission. Similar signatures can be found in b) and d), where molecular vibrations are turned off, but only in the first peak - here, on the longer time scale, the dephasing from the higher-level excitations of the cavity mode leads to slightly shifted maxima for increasing $N$ (slightly different time scales of recurrence) and finally to a loss of coherence. 

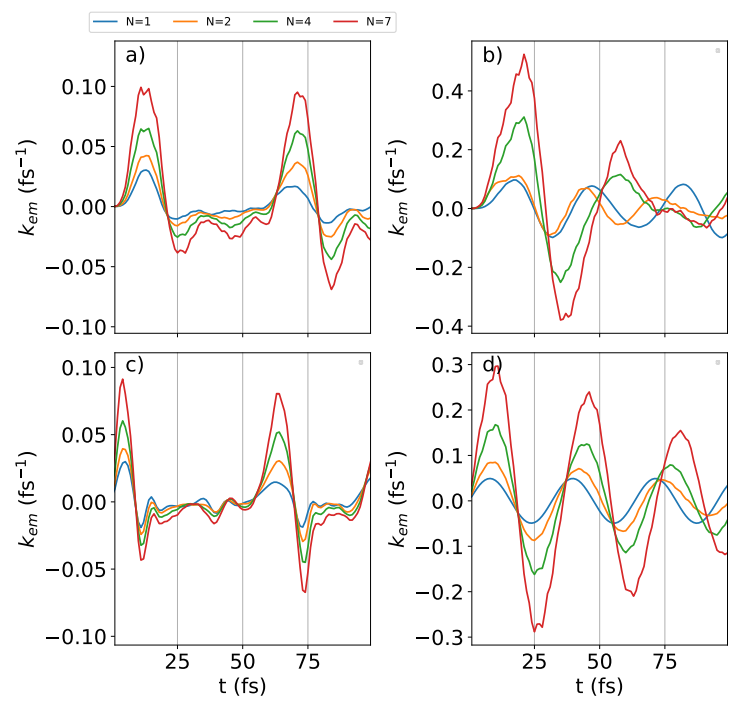

FIG. 3. The pyrazine model with the IC channel closed, emission rate. Excitation with a) laser pulse, b) laser pulse (no vibrational tuning in the molecule), c) instantaneous excitation, d) instantaneous excitation (no vibrational tuning in the molecule).

\section{B. Pyrazine ensemble coupled to the cavity via the $S_{0}-S_{2}$ transition}

We now consider pyrazine in a cavity resonantly coupling $S_{0}$ and $S_{2}$. The nonadiabatic transition from $S_{2}$ to $S_{1}$ competes with radiative emission into the cavity, so that either the excitation can be utilized to undergo internal conversion, resulting in the product state $S_{1}$; or, the photon is emitted into the cavity, from where excitation can be restored in the molecule through Rabi cycling. In the following, the cavity resonantly couples (i) at the FC point $\left(\omega_{C}=4.723 \mathrm{eV}\right)$; (ii) at the $\mathrm{S}_{2}$ potential minimum $\left(\omega_{C}=4.198 \mathrm{eV}\right)$. A range of laser pulses is investigated, where the integral of the laser-pulse envelope is kept constant for varying laser-pulse duration. The pulse parameters are selected to achieve a roughly $50 \%$ excitation of one molecule; the excitation frequency targets the $\mathrm{S}_{0}-\mathrm{S}_{2}$ transition at the FC point with $\omega_{L}=4.723 \mathrm{eV}$, and the intensities are given in Table I.

The onset of emission, apparent in the peaks of the emission rate, is shown in Fig. 4. Transient negative emission rates signify re-absorption of photons by the molecules. Generally, the emission rate is higher for resonance at the FC point (left panel) than resonance at the $S_{2}$ potential minimum (right panel). In case of an instantaneous excitation, a) to d), the onset of emission occurs much earlier, whereas it is shifted towards later times in case of the laser-pulse excitation, e) to h). The emission rate exhibits a strong dependence on the number of molecules in the cavity in all cases; especially for the $50 \%$ instantaneous excitation and the long laser pulse for resonance at the FC 
Multiphoton excitation of a cavity-molecular ensemble

TABLE I. Instantaneous excitation, laser pulse parameters and Figure references for the different laser pulses used to excite the pyrazine molecules. One cycle FWHM corresponds to $\approx 0.875$ fs pulse duration, FWHM.

resonance at $\mathrm{FC}$ point

resonance at $\mathrm{S}_{2}$ minimum

\begin{tabular}{ccccc} 
FWHM (fs) [cycles] & intensity $\left(\mathrm{W} / \mathrm{cm}^{2}\right)$ & Fig. & intensity $\left(\mathrm{W} / \mathrm{cm}^{2}\right)$ & Fig. \\
\hline $0(100 \%$ exc.) $[0]$ & - & $4 \mathrm{a}), 5 \mathrm{a})$ & - & $4 \mathrm{~b}), 5 \mathrm{~b})$ \\
$0(50 \%$ exc.) $[0]$ & - & $4 \mathrm{c}), 5 \mathrm{a})$ & - & $4 \mathrm{~d}), 5 \mathrm{~b})$ \\
$1.75[2]$ & $4.30 \cdot 10^{13}$ & $4 \mathrm{e}), 5 \mathrm{a})$ & $2.19 \cdot 10^{13}$ & $4 \mathrm{f}), 5 \mathrm{~b})$ \\
$3.50[4]$ & $1.08 \cdot 10^{13}$ & $5 \mathrm{a})$ & $5.48 \cdot 10^{12}$ & $5 \mathrm{~b})$ \\
$5.25[6]$ & $4.78 \cdot 10^{12}$ & $5 \mathrm{a})$ & $2.44 \cdot 10^{12}$ & $5 \mathrm{~b})$ \\
$7.00[8]$ & $2.69 \cdot 10^{12}$ & $5 \mathrm{a})$ & $1.37 \cdot 10^{12}$ & $5 \mathrm{~b})$ \\
$8.75[10]$ & $1.72 \cdot 10^{12}$ & $4 \mathrm{~g}), 5 \mathrm{a})$ & $8.77 \cdot 10^{11}$ & $4 \mathrm{~h}), 5 \mathrm{~b})$ \\
\hline \hline
\end{tabular}

point, c) and g); and for $100 \%$ excitation and the short laser pulse for resonance at the $\mathrm{S}_{2}$ minimum, b) and f). In the cases where the excitation takes place instantaneously, the rate at time zero is independent of the number of molecules for $100 \%$ excitation, whereas it scales with $N$ (indicating SR) for $50 \%$ excitation. It is also interesting to note that the emission characteristics in b), d) and f) are similar, as the $S_{0}-S_{2}$ coherence is built up over time and away from the FC geometry, and hence the emission characteristics are less sensitive to the initial excitation probability. In the case of $h$ ), the build-up of coherence is prevented through the long laser pulse that leads to a more continuous population of the resonance region rather than simultaneous.

The scaling of the emission rate over $N$ for the peaks marked in Fig. 4 is shown in Fig. 5 for the different laser-pulse lengths and for instantaneous excitation as referenced in Table I. For instantaneous excitation the scaling at time $t=0$ is also shown. In a), the cavity is resonant at the FC point; and the relative emission rate exhibits a linear dependence on $N$ for all cases except the two-cycle pulse and $100 \%$ excitation at $t=0$, where the rate remains (almost) constant over $N$ and does not carry a signature of SR. With increasing pulse duration (number of cycles), the rate increase over $N$ also increases. This is due to the smaller bandwidth, which, instead of creating a broad vibrational wavepacket that quickly leaves the FC region, creates a narrower distribution of vibronic states from which coherence with the cavity can build up. The instantaneous 50\% excitation breaks this trend and, at $t=0$, has a similar scaling as the longer pulses with 8 and 10 

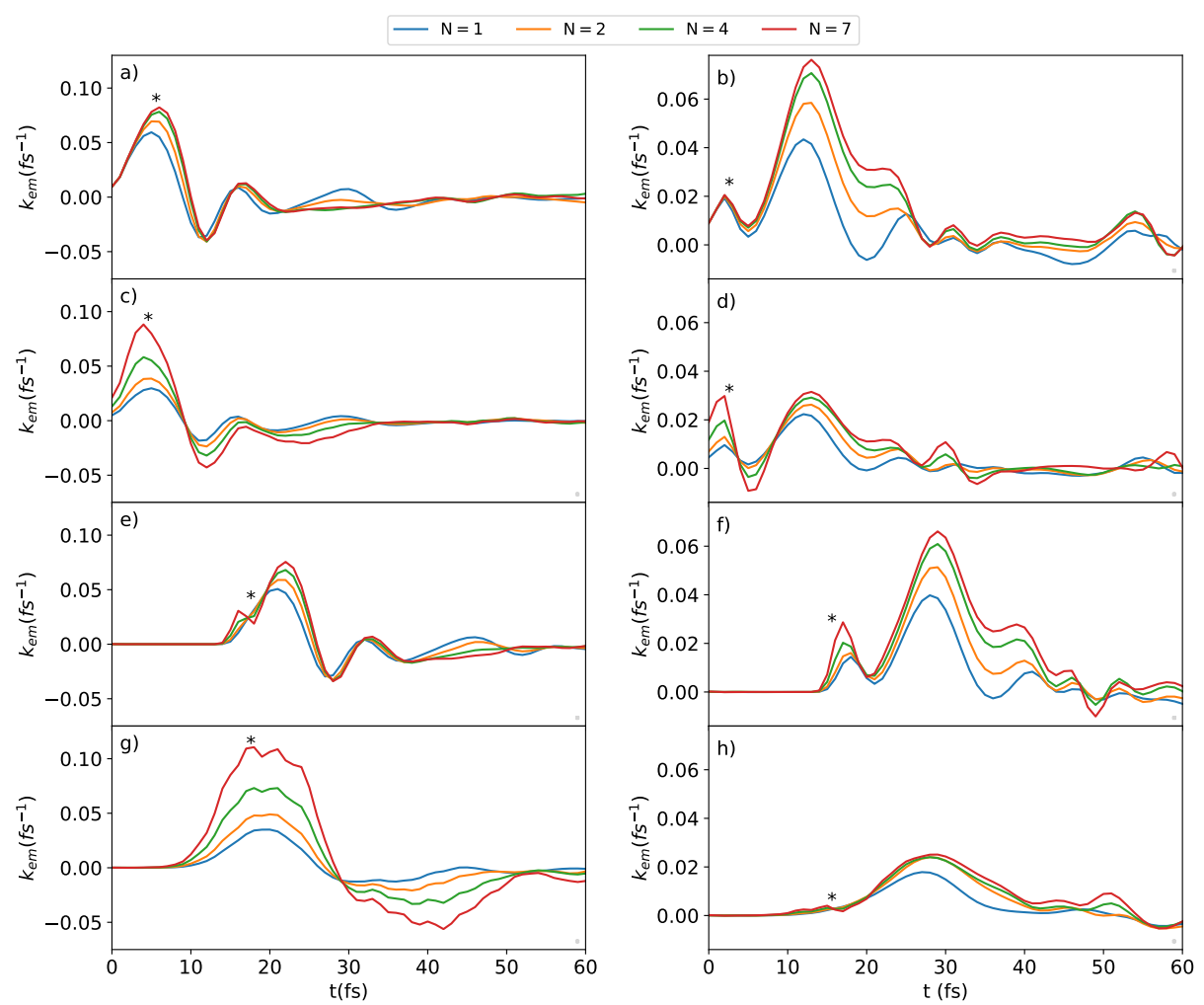

FIG. 4. Emission rate of $S_{0}-S_{2}$ resonance at the FC point (left panel) and at the $S_{2}$ minimum (right panel), with $100 \%$ instantaneous excitation (a), b)), 50\% instantaneous excitation (c), d)), the two-cycle laser pulse (e), f)) and the ten-cycle laser pulse (g), h)). The asterisk marks the time value used for the determination of the rate scaling over $N$.

cycles. The scaling with $N$, however, substantially decreases already after just $2 \mathrm{fs}$, as soon as the wavepacket moves from the FC region. The instantaneous $100 \%$ excitation forms the limiting case with no scaling of the emission rate with $N$ at $t=0$.

In Fig. 5b), the cavity resonance lies at the $S_{2}$ minimum configuration. As expected, the $100 \%$ excitation leads to no SR and a constant rate over $N$ at time $t=0$, but in this case also for the later peak at $t=6 \mathrm{fs}$. For the $50 \%$ instantaneous excitation, SR is observed both at $t=0$ and for the later peak at $t=2 \mathrm{fs}$. Overall, the opposite trend as in a) is observed, with the shortest laser pulse resulting in the largest rate increase; while all laser pulses result in SR characteristics. The reason for this opposite behaviour compared to resonance at the FC point lies in the smaller temporal width of the excited wave packet due to the shorter pump pulse - the wave packet is less spread out in time, and evolves more coherently on the excited state.

There is a peak at a later time in Fig. 4 (right panel) that exhibits different rates for different 
Multiphoton excitation of a cavity-molecular ensemble

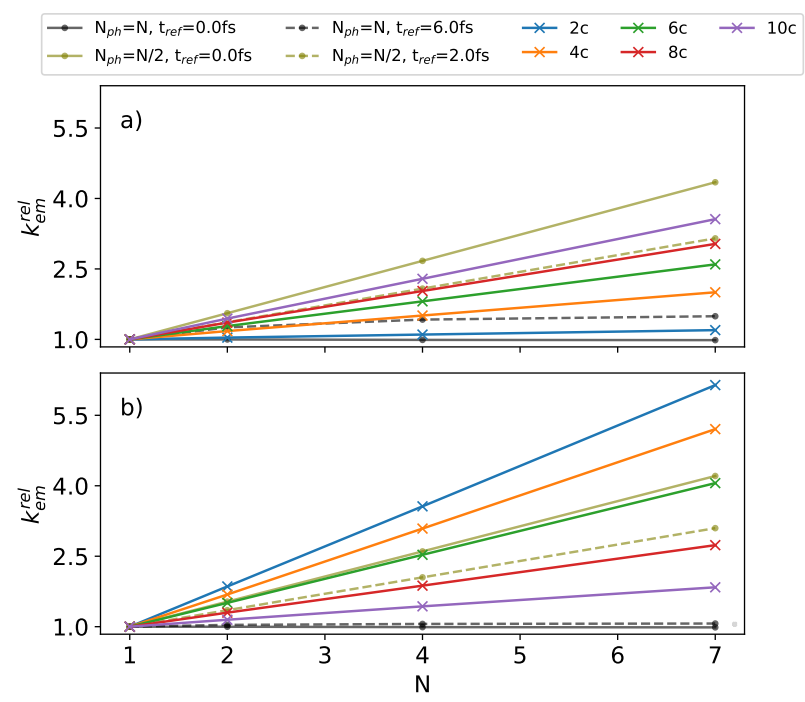

FIG. 5. Scaling of the relative emission rate constant over $N$ for $\mathrm{S}_{0} \mathrm{~S}_{2}$ resonance at a) the FC point and b) the $\mathrm{S}_{2}$ minimum.

ensemble sizes; this peak is present in all of the calculations for resonance at the $S_{2}$ minimum, and also resembles SR, but the rate increase seems to flatten off towards a constant value for larger ensemble sizes. This reemergence of SR over time has also been found in the previous section for the molecule with the IC channel closed, and occurs when there is a roughly 1:1 population of $\mathrm{S}_{2}$ and $\mathrm{S}_{0}$ (see $\mathrm{SI}$ ). But as the scaling of the rate constant depends on the overlap between ground and excited state (cf. Eq. (6)), the rate increase flattens off with increasing ensemble size due to the overlap factor. Only in the case of $50 \%$ excitation and the very short laser pulses is the linear scaling retained also up to seven molecules.

Previously ${ }^{29}$ we had shown that the collective effects diminish quickly for one-photon excitation with a cavity resonantly coupling two states for a configuration far from the FC point. The molecules that are not excited, and that could partake in the cavity-ensemble interaction, mostly reside in the FC region in configuration space. For a multiphoton excitation, the situation is reversed with the excited molecules mostly populating the minimum of the excited state and not the FC point. Thus, collective effects will be more visible for a cavity coupling at the $S_{2}$ minimum and not at the FC point, and this is reflected in the much more pronounced rate enhancement over $N$ in Fig. 5b) compared to a).

The effect of the laser-pulse duration also largely depends on which region in configuration space is resonant with the cavity. For a resonance at the FC point, it is advantageous to use a 
Multiphoton excitation of a cavity-molecular ensemble

narrow-band laser pulse (small frequency spread but longer duration). Through such a pulse, population is transferred to a smaller group of vibronic states, from which coherence with the cavity can build up, finally resulting in a SR burst. On the other hand, for a cavity resonant with the $S_{0}-S_{2}$ gap at the $S_{2}$ minimum, SR is enhanced if the wavepacket is created by a shorter pulse and hence has a narrower time-distribution, as it reaches the resonant region with the cavity at once.

Recently, it has been shown theoretically that the time-dependent emission of a low- $Q$ cavity can be used to track the intramolecular dynamics after excitation of the ensemble. ${ }^{36}$ It was demonstrated that the motion of the nuclear density in and out of the resonance region can be followed through the emission characteristics, as an emission requires the population of polaritonic modes, which are local probes of the molecular configuration. Although, in both studied situations, emission occurs quite obviously whenever the nuclear wavepacket is found at a geometry resonant with the cavity, in Ref. 36 no SR enhancement in the emission rate is observed. The reason for this is the fact that the laser pulse excites the system in the perturbative, one-photon regime, in which SR cannot take place.

These trends highlight the implications for the emergence of long-lived vibronic coherence in systems that are excited either with broad-band or narrow-band laser pulses, and for laboratory experiments compared, e.g., to sunlight absorption. ${ }^{24}$ Different excitation mechanisms and the degree of initial coherence clearly initiate different dynamics, whose coherence can be probed by the emission characteristics to a cavity mode coupled to the ensemble.

\section{Resonance between $S_{0}$ and $S_{1}$ : Frequency downconversion}

Turning to the third scenario - resonance of the cavity with the $S_{0}-S_{1}$-transition - the process of laser-pulse excitation of the molecule leading to an emission into the cavity can be seen as a frequency down-conversion from $4.72 \mathrm{eV}(260 \mathrm{~nm})$ to $3.80 \mathrm{eV}(326 \mathrm{~nm}$, resonance at the FC point) or $3.50 \mathrm{eV}\left(354 \mathrm{~nm}\right.$, resonance at the $\mathrm{S}_{1}$ potential minimum). If the radiation is emitted in an SR burst, it will be highly coherent and directional. However, the requirement for such an emission is that the wave packet after excitation evolves coherently among the different molecules, and that coherence is preserved between the different vibronic states even after the IC.

As discussed above, collective effects for a resonance of $S_{0}$ and $S_{1}$ at the $S_{1}$ potential minimum are very small for single-photon excitation, as there is almost no density in the $\mathrm{S}_{0}$ state at the 
Multiphoton excitation of a cavity-molecular ensemble

configuration of the $\mathrm{S}_{1}$ minimum ${ }^{29}$. However, collective effects persist for a resonance at the FC point, as there is sufficient density in the ground state. For a multiphoton excitation, the situation is reversed: There is a larger collective effect for a resonance at the $S_{1}$ minimum because many molecules reach this geometry at similar times and coherently re-populate $S_{0}$ at this geometry through the cavity.

A condition for the emergence of SR is a coherently evolving wave packet in the excited state between different members of the ensemble. A second requirement for SR is that there is also density in the ground state vertically below the excited state, and that the ground-state wave packet of each molecule is coherent with the corresponding excited-state, which relates back to the overlap of the vibronic wavepackets in the electronic states $S_{1}$ and $S_{0}$. In the following, a $100 \%$ instantaneous excitation of the molecules is initiated at the FC point as previously; so that each molecule is excited into its $\mathrm{S}_{2}$ electronic state at the same time. The wave packet then passes through the conical intersection and evolves toward the $S_{1}$ minimum configuration. Along the pathway, and close to the $S_{1}$ minimum, radiation can be emitted into the cavity. However, the emitted radiation will initially not show any dependence on $N$ (recall, we scale the molecule-cavity interaction by a factor $1 / \sqrt{N}$ ), as there will be no coherent population in $\mathrm{S}_{0}$; however, after a period of slow incoherent emission, coherent population in the ground state will build up, leading to the emergence of SR.

In Fig. 6a), the emission rate over time and b) the scaling of the relative emission rate over $N$ for selected instances in time is shown for $100 \%$ excitation and resonance at the FC point. A first increase of the emission rate is observed around $18 \mathrm{fs}$, when the first part of the wave packet has undergone IC and populated the $\mathrm{S}_{1}$ state. This peak shows no SR signature apparent from the almost constant relative emission rate over $N$ for $t_{\text {ref }}=18 \mathrm{fs}$. With more and more emission into the cavity, a resonance between $S_{1}$ and $S_{0}$ vibronic states can manifest and lead to the peak at $32 \mathrm{fs}$ exhibiting a rate increase for increasing $N$. The relative emission rate in $\mathrm{b}$ ) for this peak exhibits only sublinear scaling, but is also not constant over $N$. The additional marked peaks in the emission rate a) exhibit similar features, though the rate increase is lower. From the sublinear scaling over $N$, it is seen that the rate increase levels off quickly towards a constant value. Thus, we can determine that there is a partial build-up of electronic coherence between the $S_{0}$ and $S_{1}$ electronic states as they become coupled through the cavity. This is quite remarkable, as $S_{1}$ is reached only after the system has decayed through the conical intersection between $S_{2}$ and $S_{1}$, which results in a fairly broad nuclear wavepacket. According to Eq. 6, the enhancement of the 
Multiphoton excitation of a cavity-molecular ensemble
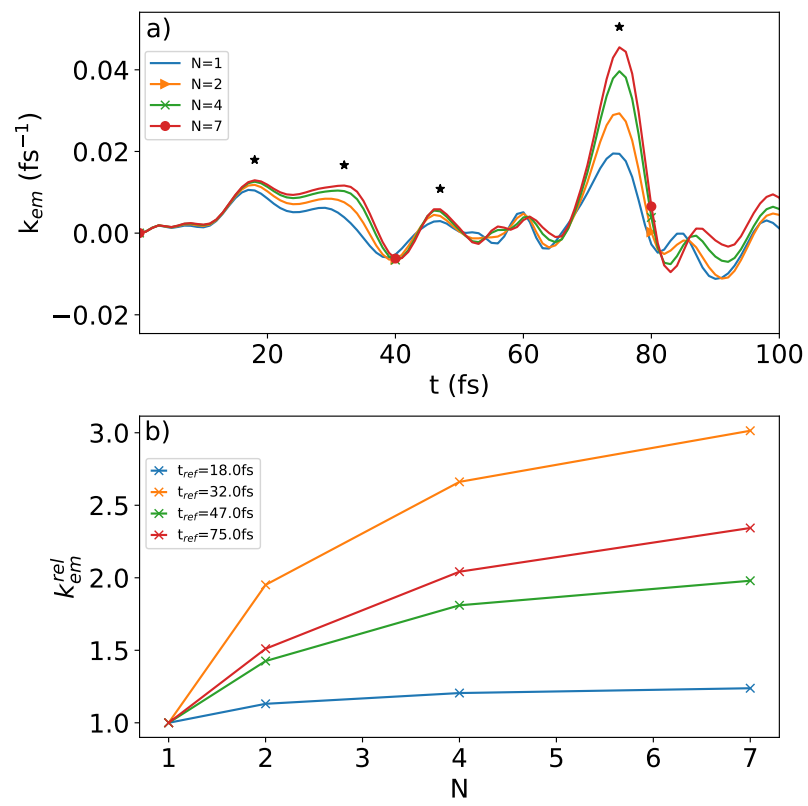

FIG. 6. $\mathrm{S}_{0}-\mathrm{S}_{1}$ resonance at the FC point, a) absolute and b) relative emission rate. The peaks of the emission rate marked in a) are used to determine the scaling in b) over the number of molecules $N$.

emission rate as a function of $N$ is a direct proxy of this coherence. Nonetheless, the SR emission flattens already between 4 and 7 molecules, indicating that the build-up of coherence is partial and does not extend to the whole ensemble. We also studied different laser pulses but in this case the behaviour is similar to $100 \%$ excitation and hence much less sensitive to the initial preparation.

In Fig. 7a), the transient emission rate and b) the scaling of the relative emission over $N$ for selected time-delays is shown, both for $100 \%$ excitation and resonance at the $\mathrm{S}_{1}$ minimum. Similar features as for resonance at the FC point are observed. However, the rate scaling is significantly higher with a seven-fold increase for the peak at $84 \mathrm{fs}$. This is consistent with more pronounced collective effects for the selected resonance. However, also in this case the SR behaviour levels off and an extension of the coherence to the whole ensemble is not to be expected for larger ensemble sizes. Nonetheless, the emission rate increase is still significant even after passage through the CI; where selecting the cavity resonance at the geometry of a local minimum of the upper PES leads to a much more pronounced emission rate enhancement over increasing ensemble size. 
Multiphoton excitation of a cavity-molecular ensemble
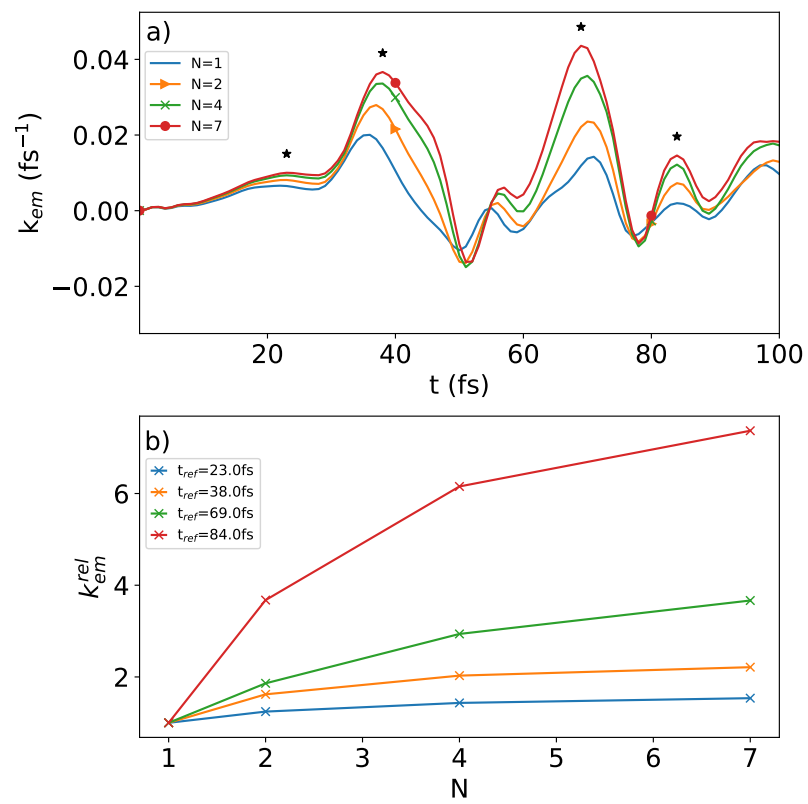

FIG. 7. $S_{0}-S_{1}$ resonance at the $S_{1}$ potential minimum, a) absolute and b) relative emission rate. The peaks of the emission rate marked in a) are used to determine the scaling in b) over the number of molecules $N$.

\section{SUMMARY}

The signature of SR emission in molecular ensembles is linear in a regime of constant emitter density. This signature has been investigated for a model of a molecular system featuring an internal conversion channel, in terms of the ensemble excitation mechanism and the position of the cavity-molecule resonance. It has been shown that a $50 \%$ instantaneous excitation of the $\mathrm{S}_{2}$ state of all ensemble members leads to SR emission on the ultrashort and short time scale, while for a $100 \%$ excitation of the ensemble the SR signature emerges later, and the emission rate scales less favorably with increasing number of emitters. The excitation with a laser pulse also affects the SR characteristics, in dependence on the position of the cavity-molecule resonance. For a resonance at a nonstationary point in the electronic excited potential energy surface, a narrowband laser pulse selectively targeting the excitation leads to more coherent emission. Instead, if the cavity is resonant at the geometry of a local minimum in the upper potential, a shorter pulse with a broader bandwidth results in a more coherent emission of light. These observations have important implications for light harvesting, as the arguments can be translated to the differences in the excitation mechanism by depending on the coherence properties of the light source, which directly impact the coherence and emission properties. ${ }^{24}$ The SR emission can also be utilized 
in frequency conversion, as demonstrated by employing the internal conversion of the pyrazine molecules as a downconverter. A vibronic coherence build-up between $S_{1}$ and $S_{0}$ is observed, that results in SR emission even after passage through the CI. This coherence is more pronounced for a cavity resonant with the $S_{1}$ minimum compared to the FC point. Overall, SR signatures are prevalent in the multiphoton excitation of cavity-molecular ensembles and provide a window to observe vibronic coherence in macroscopic systems.

\section{ACKNOWLEDGMENTS}

This research was supported by the Deutsche Forschungsgemeinschaft (DFG) Grant Nr. VE922/1-1.

\section{SUPPORTING INFORMATION}

See Supporting Information for cavity-mode populations for the pyrazine model with no IC channel; and electronic populations for pyrazine with the cavity coupling $S_{0}$ and $S_{2}$ for the different excitation mechanisms.

\section{DATA AVAILABILITY STATEMENT}

The data that support the findings of this study are available from the corresponding author upon reasonable request.

\section{REFERENCES}

${ }^{1}$ M. Hertzog, M. Wang, J. Mony, and K. Börjesson, "Strong Light-Matter Interactions: A New Direction within Chemistry," Chem. Soc. Rev. 48, 937 (2019).

${ }^{2}$ R. H. Dicke, “Coherence in spontaneous radiation processes,” Phys. Rev. 93, 99-110 (1954).

${ }^{3}$ J. C. Macgillivray and M. S. Feld, "Superradiance in atoms and molecules," Contemporary Physics 22, 299-810 (1981).

${ }^{4}$ M. Gross and S. Haroche, "Superradiance: An essay on the theory of collective spontaneous emission,” Physics Reports 93, 301-396 (1982). 
Multiphoton excitation of a cavity-molecular ensemble

${ }^{5}$ L. I. Men'shikov, "Superradiance and related phenomena," Physics-Uspekhi 42, 107-147 (1999).

${ }^{6}$ V. V. Temnov and U. Woggon, "Superradiance and Subradiance in an Inhomogeneously Broadened Ensemble of Two-Level Systems Coupled to a Low-Q Cavity,” Phys. Rev. Lett. 95, 243602 (2005).

${ }^{7}$ S. Lukyanetsa and D. Bevzenko, "Enhanced Superradiance Effect in a System of Interacting Two-Level Atoms and Crossover from Coherent to Many-Atom Multiphoton Relaxation Regime," Optics and Spectroscopy 111, 727-732 (2011).

${ }^{8}$ O. Vendrell, "Coherent Dynamics in Cavity Femtochemistry: Application of the MultiConfiguration Time-Dependent Hartree Method," Chem. Phys. 509, 55-65 (2018).

${ }^{9}$ J. G. Bohnet, Z. Chen, J. M. Weiner, D. Meiser, M. J. Holland, and J. K. Thompson, “A steadystate superradiant laser with less than one intracavity photon," Nature 484, 78-81 (2012).

${ }^{10}$ A. Goban, C.-L. Hung, J. D. Hood, S.-P. Yu, J. A. Muniz, O. Painter, and H. J. Kimble, “Superradiance for Atoms Trapped along a Photonic Crystal Waveguide,” Phys. Rev. Lett 115, 63601 (2015).

${ }^{11}$ J. Schachenmayer, C. Genes, E. Tignone, and G. Pupillo, "Cavity-Enhanced Transport of Excitons,” Phys. Rev. Lett. 114, 196403 (2015).

${ }^{12}$ B. Casabone, K. Friebe, B. Brandstätter, K. Schüppert, R. Blatt, and T. E. Northup, "Enhanced Quantum Interface with Collective Ion-Cavity Coupling,” Phys. Rev. Lett. 114, 023602 (2015).

${ }^{13}$ A. González-Tudela, V. Paulisch, H. J. Kimble, and J. I. Cirac, "Efficient Multiphoton Generation in Waveguide Quantum Electrodynamics,” Phys. Rev. Lett. 118, 213601 (2017).

${ }^{14}$ N. Skribanowitz, P. Herman, J. C. Macgillivray, and M. S. Feld, “Observation of Dicke Superradiance in Optically Pumped HF Gas,” Phys. Rev. Lett. 30, 309 (1973).

${ }^{15}$ F. C. Spano and S. Mukamel, "Superradiance in molecular aggregates,” J. Chem. Phys. 91, 683700 (1989).

${ }^{16}$ S. P. Lukyanets and D. A. Bevzenko, "Effects of interatomic interaction on cooperative relaxation of two-level atoms," Phys. Rev. A 74, 053803 (2006).

${ }^{17}$ Q. Deng and D. G. Deppe, "Spontaneous-emission coupling from multiemitters to the quasimode of a Fabry-Pérot microcavity," Phys. Rev. A 53, 1036 (1996).

${ }^{18}$ A. Auffèves, D. Gerace, S. Portolan, A. Drezet, and M. F. Santos, "Few emitters in a cavity: from cooperative emission to individualization," New Journal of Physics 13, 93020 (2011). 
Multiphoton excitation of a cavity-molecular ensemble

${ }^{19}$ S. Fuchs, J. Ankerhold, M. Blencowe, and B. Kubala, "Non-equilibrium dynamics of the Dicke model for mesoscopic aggregates: signatures of superradiance," Journal of Physics B: Atomic, Molecular and Optical Physics 49, 035501 (2016).

${ }^{20}$ V. N. Pustovit and T. V. Shahbazyan, "Plasmon-mediated superradiance near metal nanostructures,” Phys. Rev. B 82, 075429 (2009).

${ }^{21}$ Y. Luo, G. Chen, Y. Zhang, L. Zhang, Y. Yu, F. Kong, X. Tian, Y. Zhang, C. Shan, Y. Luo, J. Yang, V. Sandoghdar, Z. Dong, and J. G. Hou, "Electrically Driven Single-Photon Superradiance from Molecular Chains in a Plasmonic Nanocavity," Physical Review Letters 122, 233901 (2019).

${ }^{22}$ G.-D. Lin and S. F. Yelin, "Vibrational spectroscopy of polar molecules with superradiance," Mol. Phys. 111, 1917-1922 (2013).

${ }^{23}$ F. P. Diehl, C. Roos, A. Duymaz, B. Lunkenheimer, A. Köhn, and T. Basché, "Emergence of coherence through variation of intermolecular distances in a series of molecular dimers," J. Phys. Chem. Lett. 5, 262-269 (2014).

${ }^{24}$ L. F. Calderón and L. A. Pachón, "Nonadiabatic sunlight harvesting," Phys. Chem. Chem. Phys. 22, 12678-12687 (2020).

${ }^{25}$ G. A. Worth, H. Meyer, and L. S. Cederbaum, "The Effect of a Model Environment on the $\mathrm{S}_{2}$ Absorption Spectrum of Pyrazine: A Wave Packet Study Treating all 24 Vibrational Modes,' J. Chem. Phys. 105, 4412-4426 (1996).

${ }^{26}$ A. Raab, G. A. Worth, H.-D. Meyer, and L. S. Cederbaum, "Molecular Dynamics of Pyrazine after Excitation to the $\mathrm{S}_{2}$ Electronic State Using a Realistic 24-Mode Model Hamiltonian,” J. Chem. Phys. 110, 936-946 (1999).

${ }^{27}$ O. Vendrell, "Collective Jahn-Teller Interactions through Light-Matter Coupling in a Cavity," Phys. Rev. Lett. 121, 253001 (2018).

${ }^{28}$ I. S. Ulusoy, J. A. Gomez, and O. Vendrell, "Modifying the nonradiative decay dynamics through conical intersections via collective coupling to a cavity mode," J. Phys. Chem. A 123, 8832-8844 (2019).

${ }^{29}$ I. S. Ulusoy and O. Vendrell, "Dynamics and spectroscopy of molecular ensembles in a lossy microcavity," J. Chem. Phys. 153, 044108 (2020).

${ }^{30}$ H. D. Meyer, U. Manthe, and L. S. Cederbaum, "The Multi-Configurational Time-Dependent Hartree Approach," Chem. Phys. Lett. 165, 73-78 (1990). 
Multiphoton excitation of a cavity-molecular ensemble

${ }^{31}$ M. H. Beck, A. Jäckle, G. A. Worth, and H.-D. Meyer, "The Multiconfiguration TimeDependent Hartree (MCTDH) Method: A Highly Efficient Algorithm for Propagating Wavepackets,” Phys. Rep. 324, 1-105 (2000).

${ }^{32} \mathrm{H}$. Wang and M. Thoss, "Multilayer Formulation of the Multiconfiguration Time-Dependent Hartree Theory," J. Chem. Phys. 119, 1289-1299 (2003).

${ }^{33}$ U. Manthe, “A Multilayer Multiconfigurational Time-Dependent Hartree Approach for Quantum Dynamics on General Potential Energy Surfaces,' J. Chem. Phys. 128, 164116 (2008).

${ }^{34}$ O. Vendrell and H.-D. Meyer, "Multilayer Multiconfiguration Time-Dependent Hartree Method: Implementation and Applications to a Henon-Heiles Hamiltonian and to Pyrazine," J. Chem. Phys. 134, 044135 (2011).

${ }^{35}$ G. A. Worth, M. H. Beck, A. Jäckle, O. Vendrell, and H.-D. Meyer, The MCTDH Package, Version 8.2, (2000). H.-D. Meyer, Version 8.3 (2002), Version 8.4 (2007). O. Vendrell and H.-D. Meyer Version 8.5 (2013). Version 8.5 contains the ML-MCTDH algorithm. Current versions: 8.4.17 and 8.5.10 (2019). See http://mctdh.uni-hd.de/.

${ }^{36}$ R. E. Silva, J. del Pino, F. J. García-Vidal, and J. Feist, "Polaritonic molecular clock for alloptical ultrafast imaging of wavepacket dynamics without probe pulses," Nature Communications 11, 1423 (2020). 